
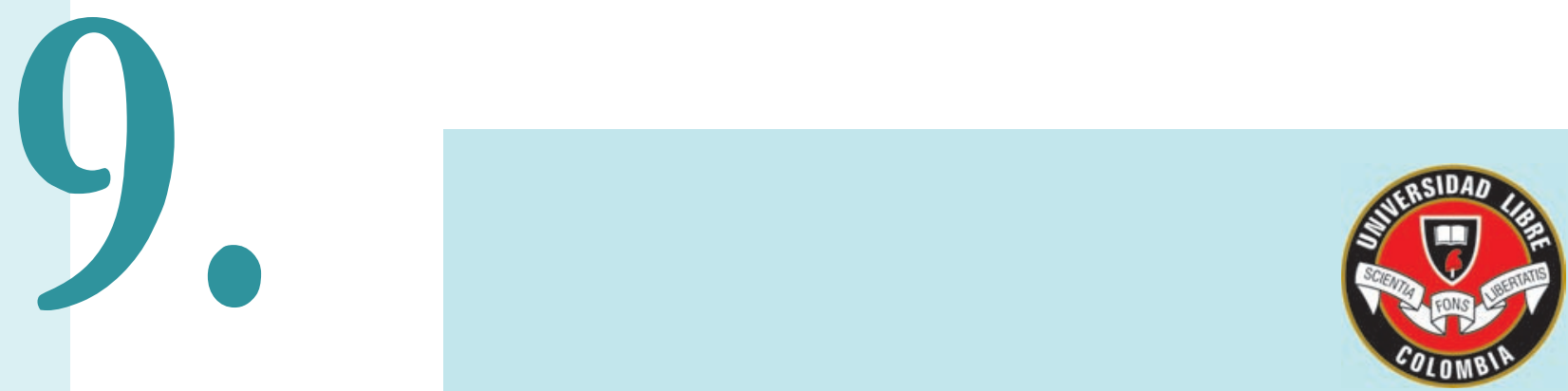

La Calidad Académica, un Compromiso Institucional

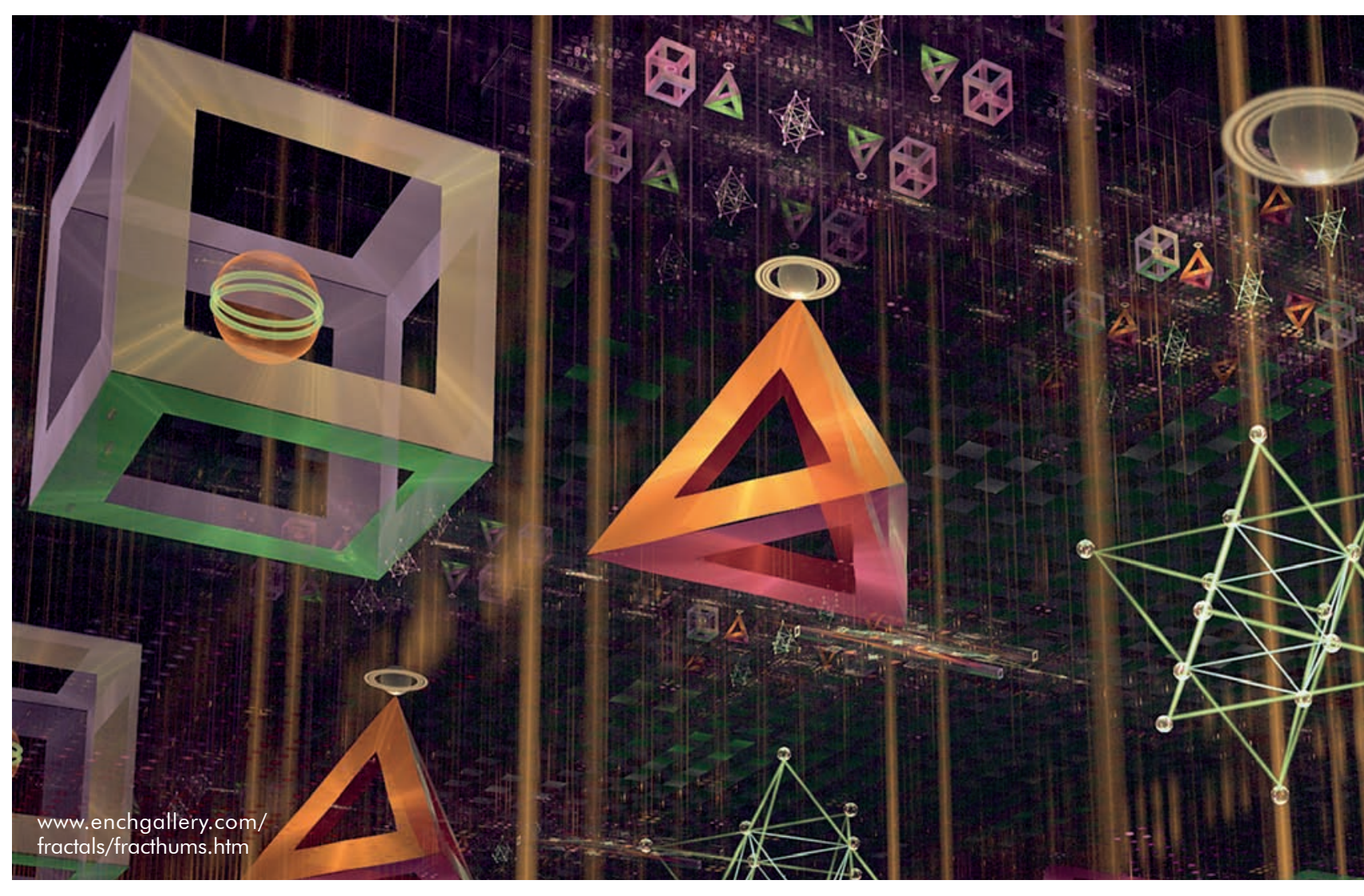

Correa M., Claudia M. y Duque O., Edison J. (2012). Evaluación del concepto de valor para el consumidor en el marketing y el diseño. Una revisión conceptual. Criterio Libre, 10 (17), 193-216

ISSN 1900-0642
Evaluación del concepto de valor para el consumidor en el marketing y el diseño. Una revisión conceptual

Claudia Marcela Correa Malagón Edison Jair Duque Oliva 


\section{EVALUACIÓN DEL CONCEPTO DE VALOR PARA EL CONSUMIDOR EN EL MARKETING Y EL DISEÑO. UNA REVISIÓN CONCEPTUAL*}

ASSESSMENT OF THE CONCEPT OF VALUE FROM A CONSUMER PERSPECTIVE IN MARKETING

AND DESIGN: A CONCEPTUAL REVISION

AVALIAÇÃO DO CONCEITO DE VALOR PARA O CONSUMIDOR NO MARKETING E O DESENHO,

UMA REVISÃO CONCEITUAL

ÉVALUATION DU CONCEPT DE VALEUR POUR LE CLIENT DANS LE MARKETING ET LE DESIGN,

UN CADRE CONCEPTUEL

\section{CLAUDIA MARCELA CORREA MALAGÓN EDISON JAIR DUQUE OLIVA§}

\footnotetext{
Artículo de investigación del análisis cualitativo del concepto de valor para el marketing y el diseño, que hace parte de las líneas de investigación de la maestría en administración, de la Universidad Nacional de Colombia.

Concept of Value in Marketing and Design analysis research, from the Masters in Business Administration Program. Universidad Nacional de Colombia.

Artigo de pesquisa da análise qualitativo do conceito de valor para o marketing e o desenho, que faz parte das linhas de pesquisa do mestrado em administração, da Universidade Nacional de Colombia.

Analyse qualitative du concept de valeur pour le marketing et le design, qui fait partie de la ligne de recherche du MBA à l'Université Nationale de Colombie.

‡ Diseñadora industrial, Universidad Nacional de Colombia; magíster en administración (C), Universidad Nacional de Colombia.cmcorream@unal.edu.co Industrial Designer, Universidad Nacional de Colombia; Masters in Business Administration (C), Universidad Nacional de Colombia.cmcorream@unal.edu.co

Desenhadora industrial, Universidade Nacional de Colombia; mestre em administração (C), Universidade Nacional de Colômbia.cmcorream@unal.edu.co

Dessinatrice industriel, Université Nationale de Colombie, Master en Management (C), Université Nationale de Colombie.cmcorream@unal.edu.co

$\S$ Administrador de empresas, Universidad Nacional de Colombia; MBA Universidad de los Andes, MSc Universitat Pompeu Fabra, DEA, PhD (C) en marketing, Universidad de Valencia; profesor, Facultad de Ciencias Económicas, Universidad Nacional de Colombia. eduqueo@unal.edu.co

Business Administrator, Universidad Nacional de Colombia; MBA Universidad de los Andes, MSc Universitat Pompeu Fabra, DEA. PhD (C) in marketing, Valencia University. Professor, Economic Science Faculty, Universidad Nacional de Colombia. eduqueo@ unal.edu.co

Administrador de empresas, Universidade Nacional de Colombia; MBA Universidade de los Andes, MSc Universitat Pompeu Fabra, DEA, PhD (C) em marketing, Universidade de Valencia; professor, Faculdade de Ciências Econômicas, Universidade Nacional de Colombia.eduqueo@unal.edu.co

Business Manager, Université Nationale de Colombie, Université des Andes MBA, MSc Universitat Pompeu Fabra, DEA, doctorat (C) en marketing, Université de Valence, Professeur, Faculté des sciences économiques, Université Nationale de Colombie. eduqueo@unal.edu.co
}

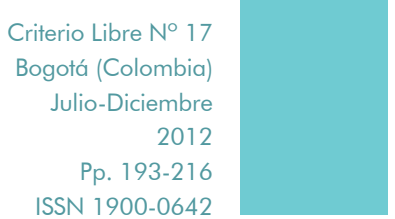




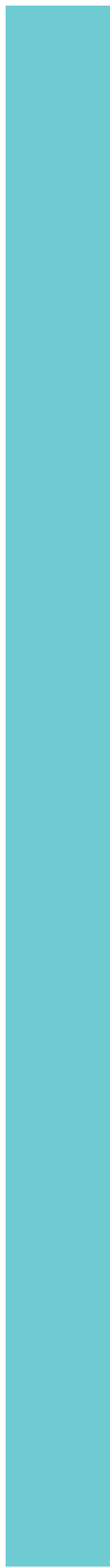

Fecha de recepción: mayo 11 de 2012

Fecha de aceptación: octubre 2 de 2012

Reception Date: May 112012

Acceptance Date: October 22012

Data de recepção: 11 de maio de 2012

Data de aceitação: 2 de outubro de 2012

Reçu le: 11 mai 2012

Accepté: Octobre 2, 2012

RESUMEN

Desde una perspectiva descriptiva y reflexiva, este artículo busca caracterizar, clasificar y analizar los conceptos de valor para el marketing y el diseño, identificando sus elementos comunes y discordantes que permitan generar una reflexión sobre su interrelación e importancia. Primero se hace un acercamiento inicial al concepto de valor, para posteriormente explorarlo desde las dos disciplinas, identificando los principales pilares conceptuales para compararlos y finalmente analizarlos en conjunto. Como conclusión, se determinó que la caracterización, identificación y clasificación de los conceptos de valor contribuye enormemente a la investigación de valor en las dos áreas y que la brecha entre estos conceptos es dada por algunas diferencias conceptuales, pero sobre todo por el grado de importancia que se le da a cada una.

\section{PALABRAS CLAVE: \\ Marketing, diseño, valor, consumidor. \\ CLASIFICACIÓN JEL: \\ M00, M31, A12.}

SUMMARY

From a descriptive and reflexive perspective, this article seeks to characterize, classify and analyze the concept of value in marketing and design, identifying their elements in common and differences and those that do not consider their interrelationship and importance. First, there is an approach to the concept of value, then an exploration of the same from both disciplines, identifying the main conceptual pillars to compare them and analyze them as a whole.

In conclusion it was determined that the characterization, identification and classification of the concept of value contributes enormously to the 
research of value in both areas and that the gap between these concepts comes from conceptual differences and especially from the importance given to each one.

Key words: Marketing, design, value, consumer. JEL Classification: M00, M31, A12.

RESUMO

A partir de uma perspectiva descritiva e reflexiva, este artigo busca caracterizar, classificar e analizar os conceitos de valor para o marketing e o desenho, identificando seus elementos comuns e discordantes que permitan gerar uma reflexão sobre sua interrelação e importância. Primeiro faz-se um acercamento inicial ao conceito de valor, para posteriormente explorálo desde as duas disciplinas, identificando os principais pilares conceituais para compará-los finalmente analizá-los em conjunto. Como conclusão, determinou-se que a caracterização, identificação e classificação dos conceitos de valor contribui enormemente à pesquisa de valor nas duas áreas e que a brecha entre estes conceitos é dada por algumas diferenças conceituais, mas sobretudo pelo grau de importância que se dá a cada uma.

Palavras chave: Marketing, desenho, valor, consumidor. Classificação JEL: M00, M31, A12.

RÉSUMÉ

À partir d'une réflexion, cet article cherche à caractériser, classifier et analyser les concepts de valeur pour le marketing et le design, l'identification des éléments communs et discordants pour générer une réflexion sur leur relation et leur importance.

Tout d'abord les auteurs font une approche à la notion de valeur, en identifiant les principaux concepts pour les comparer et les analyser à l'ensemble. En conclusion, il a été déterminé que la caractérisation, l'identification et la classification des concepts de valeur contribue à la recherche de valeur dans les deux domaines et l'écart entre ces concepts est donnée par certaines différences conceptuelles, mais surtout par le degré d'importance que l'on donne à chacun.

Mots-clés: Marketing, desing, la valeur de la consommation.

Classification JEL: M00, M31, A12.

\section{- INTRODUCCIÓN}

Desde el entendimiento popular podría afirmarse que el concepto de valor para el marketing y para el diseño debería contener conceptos cercanos 
y compatibles, debido a que son disciplinas que tienen un alto grado de relación en la práctica, dada la interconexión de sus funciones y ámbitos de aplicación. De igual manera, un diseño innovador e inteligente ayuda en la apertura de nuevos mercados, reaviva el interés del mercado en las nuevas características que les ofrece, y brinda más valor para el consumidor, reforzando los valores de la empresa a los ojos del mismo, mientras el marketing los hace conscientes de las nuevas características, bienes o servicios que se les ofrecen. Sin embargo, entre quienes hacen marketing poco se sabe acerca de las acciones y los procesos de gestión del diseño y entre quienes trabajan en diseño poco se sabe de las actividades desde una perspectiva de marketing (Bruce y Daly, 2007).

Algunos diseñadores se sienten inmunes al marketing (K. Ulrich y Eppinger, 2009), no evidenciando que esta es una relación creciente. Llompart (2009) plantea que dicha relación además de ser creciente, "se mantendrá por las necesidades que una disciplina tiene frente a la otra" (Llompart, 2009, p. 2). Este autor plantea que los ámbitos de aplicación (espacio e intereses de ambos), en los que el diseño se mueve son el mercado y la economía, por lo cual el diseño se mantiene como una acción vital para el mercado y el consumo; en palabras del autor, "estas disciplinas que nacen del seno del mercado por interés de los economistas, pasan de ser compañeras del diseño, a regir el rumbo de la acción que en ocasiones actúa como una ocupación de servicios alejada del mundo de la creación" (Llompart, 2009, p. 2).

El presente artículo busca caracterizar los conceptos de valor para las dos disciplinas, clasificándolos e identificando elementos afines y discordantes que permitan generar una reflexión sobre su interrelación y su importancia para la toma de decisiones conjuntas o individuales entre ellos. Tiene un enfoque descriptivo reflexivo de los diferentes conceptos de valor manejados desde el marketing y desde el diseño. Se desarrolla una recopilación y análisis de los mismos, partiendo de tres preguntas fundamentales: ¿̇cuál es la concepción de valor para cada disciplina, sus diferencias o similitudes? , ¿̇cuáles son los pilares conceptuales del valor y los constructos que las sustentan en el contexto de estas disciplinas? y żcuál es el grado de importancia que tienen dichas concepciones de valor para cada una?

El concepto de valor es tratado desde diferentes enfoques, con ricas y variadas definiciones, así como una versatilidad en las dimensiones de su investigación (Gil y Gallarza, 2008), demostrando que este tema continúa siendo un aspecto por desarrollarse, por lo que el problema propuesto se encuentra latente haciendo imperativo su estudio.

Tal como lo mencionan Gallarza, Gil y Hoolbrok (2011), es importante la investigación sobre el valor del consumidor debido a las considerables implicaciones epistemológicas tanto para el marketing como para el diseño, además para conectar constructos que permitan explicar las diferentes facetas del comportamiento del consumidor antes y después de la compra, así como para engranar los procesos de gestión de diseño y marketing, lo cual tiene implicaciones macro en campos como la responsabilidad social, la política y la ética (Gallarza, Gil Saura y Holbrook, 2011). De igual manera, la importancia de tratar estas dos visiones radica en la práctica real empresarial; no es claro el concepto de valor para ninguna de las dos disciplinas, lo que de cierta manera difiere en el modo como es aprovechada y concebida su interrelación.

La existencia de paradigmas diferentes en cuanto a la concepción y ponderación del valor ha generado una desarticulación y, para algunos, un rechazo entre las disciplinas; por consiguiente no se desarrollan estrategias que atiendan efectivamente las expectativas y los deseos de consumo de sus clientes, así como tampoco se "venderán historias" coherentes con el producto (objetivo primordial del diseñador/proyectista), y mucho menos se creará una conexión entre la demanda del producto y la satisfacción de la experiencia final del cliente. 
${ }^{66}$ Si bien el diseñador debería ser el especialista en crear valor, las percepciones del consumidor pueden diferir en gran medida por la apreciación de este valor debido a preconcepciones como los grados de afinidad, referencias contextuales $y$ económicas, o bien al mismo becho de buscar un factor de diferenciación frente al consumidor o usuario.
Es claro que la importancia de comprender la valoración para el diseño y para el marketing es el impacto que generará, ya que este se reflejara en la intención de compra del cliente final, lo cual es de vital trascendencia para las dos disciplinas. Asimismo, el hecho de combinar el diseño y el marketing sinérgicamente busca integrar conocimientos en pro del beneficio mutuo de las disciplinas, generando un trabajo académico que, de ser posible, tenga aplicación en los dos marcos y a futuro tenga mayores contribuciones investigativas. De igual manera, es clave propiciar y socializar la importancia del diseño como parte fundamental de la organización, por lo que debe propagarse en el interior de esta para generar valor y experiencia satisfactoria para el consumidor (Brown, 2009).

El punto de partida de esta revisión se basa en que el desarrollo del concepto de valor en marketing ha sido revisado y perfeccionado, pero es un tema complejo con múltiples dificultades conceptuales, metodológicas y de medición, manteniendo su pertinencia y un alto potencial investigativo (Gallarza, et al., 2011). Por otro lado, en el diseño es un tema algo olvidado, por lo cual no se encuentran muchas referencias y menos aún investigaciones. Esto hace indispensable su estudio debido a su importancia intrínseca. De este modo se hace un acercamiento inicial al concepto de valor, para posteriormente explorar dicho concepto desde el marketing y desde el diseño, identificando los principales pilares conceptuales de cada disciplina para compararlos entre sí, basándonos en su definición, clasificación e interconexión, para finalmente analizarlos en conjunto.

\section{ANÁLISIS INICIAL SOBRE}

Dada la interconexión entre las disciplinas, sería coherente inferir que el concepto de valor debería tener constructos similares, axiológicamente hablando, pero la falta de comunicación armónica y el desconocimiento entre estas disciplinas direcciona su desarrollo de maneras diversas. 
Si bien el diseñador debería ser el especialista en crear valor, las percepciones del consumidor pueden diferir en gran medida por la apreciación de este valor debido a preconcepciones como los grados de afinidad, referencias contextuales y económicas, o bien al mismo hecho de buscar un factor de diferenciación frente al consumidor - usuario. De igual manera, el diseñador debería desarrollar un papel fundamental para el marketing, siendo el encargado de visualizar el concepto del producto en forma integral, evidenciando lo que se quiere conseguir, a quién va dirigido el producto, los beneficios que aportará al usuario, cómo y cuándo se usará el producto, a qué nivel de precios debe situarse en el mercado, con qué categoría de productos debe identificarse o diferenciarse y qué relación debe tener con los otros productos de la empresa (Montaña y Fundación, 2001), agregando sentimientos estéticos, intelectuales, simbólicos, culturales, de autoestima y de pertenencia. Esto muestra cómo el diseño desempeña un papel especial en la definición de aquellos elementos funcionales y sensoriales menos evidentes, más relacionados con el significado y, por tanto, más difíciles de medir o acotar.

En general, el concepto de valor ha sido abordado desde múltiples perspectivas. Por un lado, para el marketing el valor es más un concepto relacionado con el intercambio, la utilidad y el trabajo, sin dejar de lado sus raíces psicológicas (Payne y Holt, 2001), mientras que para el diseño el valor se denota bajo 7 columnas: la acción humana, la prospectiva, la innovación, la relación cuerpo/espacio, la eficiencia, la comunicación y la interacción (Bonsiepe, 1993).

Básicamente para algunos diseñadores el marketing es una herramienta para comunicar o para dar cierta comprensión del mercado, pero no es desarrollado a fondo y menos aún desplegado adecuadamente a lo largo del proceso de diseño, mientras que para el marketing el diseño podría considerarse solo un estilizador en el diseño y desarrollo de producto, obviando su real importancia y generando preconcepciones sesgadas que limitan la real interacción entre las dos disciplinas.

\section{CONCEPTO DE VALOR DESDE EL MARKETING}

La palabra valor se encuentra pobremente diferenciada de otros conceptos como "utilidad", "precio", "calidad" y "satisfacción" (SánchezFernández e Iniesta-Bonillo, 2006), por lo que pueden surgir muchas equivocaciones y malentendidos conceptuales. Para algunos autores, el concepto de valor se ha convertido en la expresión más "sobreusada", ya que términos como "valor juzgado", "valor para el consumidor", "valor percibido" o "valor de compra", entre otros, son mal aplicados en las ciencias sociales en general y en la literatura de management en particular (Holbrook, Sánchez e Iniesta, 2009), lo cual obstaculiza su adecuada investigación.

Uno de los enfoques de la investigación sobre el valor aborda el concepto desde la perspectiva de transacción y de adquisición (Monroe, 1979), muy relacionado con la noción de utilidad y de origen microeconómico. Por otro lado está el enfoque utilitarista, psicológico y hedonista (Holbrook y Hirschman, 1982), que agrupa la concepción tradicional de funcionalidad con aspectos sensoriales, como muestra la Tabla 1.

Por otro lado, en la Figura 1 pueden apreciarse otros enfoques investigativos y sus más importantes aplicaciones dentro del marketing, donde se incluye el enfoque filosófico y del management organizacional a los dos ya mencionados.

Debido a la necesidad de agrupar e identificar su relevancia, a continuación se presentaran los diferentes conceptos de valor explorados, ordenados según su grado de importancia determinado por la reiteración e incidencia 
Tabla 1. Enfoques de la investigación de valor.

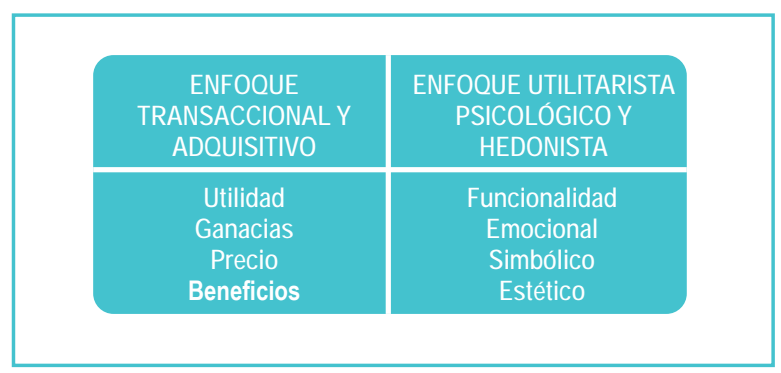

Figura 1. Orígenes y aplicaciones de la investigación de valor.

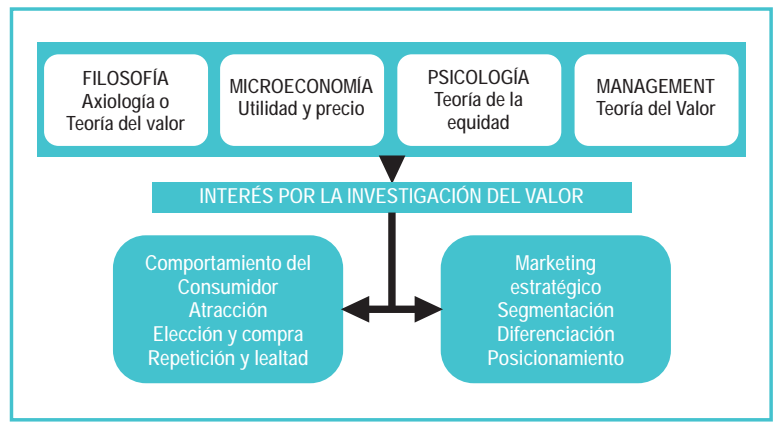

Fuente: Gil y Gallarza, 2008.

de estos en los artículos examinados para esta revisión.

Esta exploración evidencia que el principal concepto que se ha manejado del valor está relacionado con el valor de intercambio (Grönroos, 1997b), que como su nombre lo indica, es la diversa apreciación de las cosas que se da como resultado de la comparación del grado de apetencia, escasez, utilidad y satisfacción que produzca. Después, gracias a las investigaciones de autores como Kotler (1972), Bagozzi (1975), Seth, Garner y Garret (1988) Bowman y Ambrosini (1998), entre otros, se desarrollaron nuevos aspectos por tener en cuenta con relación a la maximización de la satisfacción dada por un producto, surgiendo la diferenciación de los conceptos del valor como: del producto, para el consumidor, para el cliente y el de las relaciones.

Según Holbrook (1996), el valor para el consumidor es determinado como un factor estratégico en el management, muchas veces relacionado a la creación y entrega de un valor superior, siendo sinónimo de términos como calidad y satisfacción. También constituye una herramienta importante en el análisis del comportamiento del consumidor y es parte vital del desarrollo de las relaciones a largo plazo. (Holbrook, 1996). También este autor plantea que las necesidades del consumidor, el valor percibido, su desarrollo conceptual y los métodos de medición son variables importantes en la necesidad de establecer un cuadro conceptual claro que permita una aproximación que rompa la heterogeneidad del concepto de valor (Holbrook et al., 2009).

Dentro del valor del consumidor han aparecido muchos modelos e investigaciones que han permitido un análisis más complejo e integrado. Por un lado Holbrook y Hirschman (1982), así como Zeithaml (1988) han relacionando el concepto de valor a estímulos ambientales, donde incluyendo influencias propias del consumidor, genera un sistema de comportamiento, sentimientos y conocimiento consecuente con los criterios, el aprendizaje y las consecuencias de las influencias. De acuerdo con los autores las influencias que afectan este sistema son: los recursos con que cuenta, sus capacidades de asociación y respuesta, sus actividades cotidianas, y sus características particulares que lo diferencian de los demás (Holbrook y Hirschman, 1982; Zeithaml, 1988).

Por otro lado, la medición de valor para el cliente se relaciona con el conocimiento aceptado colectivamente, más exactamente con la concepción de valor en el mercado en donde aparecen modelos que brindan información, explicación y clasificación de lo que más valoran los clientes; entre estos encontramos el valor monetario visto desde una perspectiva técnica, económica, del servicio y de los beneficios sociales que el cliente puede recibir a cambio del precio que paga (Anderson y Narus, 1998; Slater y Narver, 1994). También autores como Parasuraman (1997), Turnbull (2009) y Woodruff (1997), explican que la percepción de valor al cliente es central para determinar su satisfacción 
y lealtad, aspecto de gran importancia para cualquier organización (Parasuraman, 1997; Turnbull, 2009; Woodruff, 1997).

Un concepto asociado, el valor del cliente a largo plazo se ha venido desarrollando para mantener a los clientes y las relaciones, buscando una personalización de la experiencia del cliente, además de una completa maximización por medio de modelos de medición de estas interacciones (Berger y Nasr, 1998; Borle, Singh y Jain, 2008; Gupta et al., 2006; Jain y Singh, 2002; Venkatesan y Kumar, 2004).

El valor relacional intenta ir más allá de solo mantener a los clientes; busca proveer un único valory sostenerlo en el tiempo, incluyendo también la calidad y el servicio al cliente, eliminando la brecha entre lo que los clientes esperan y lo que reciben (Berry, 1995; Christopher, 1991; Gil y Molina, 2009; Grönroos, 1997a, 2004; Morgan y Hunt, 1994; Ravald y Grönroos, 1996; J. Sheth y Parvatiyar, 1995); por consiguiente, otro concepto de valor asociado sería el valor relacional esperado, el cual demanda un entendimiento de las relaciones de valor con la firma, y de mantener al día el acceso al valor, cuyo propósito es la construcción de relaciones de valor esperadas con relación a la probabilidad de distribución del valor presente neto y los resultados de las futuras relaciones (Glosten, Jagannathan y Runkle, 1993; Hogan, 2001).

Otro valor usado frecuentemente es el valor del servicio, el cual inicia con la medición de la calidad del servicio y la investigación de la satisfacción, donde el interés es generar un constructo práctico que se relacione con los cambios estratégicos de paradigma, generando de este nuevos conceptos afines como: calidad total, los grados de satisfacción del cliente y la gestión del valor del cliente (Cronin Jr., Brady y Hult, 2000; Mele, 2007; Parasuraman, Berry y Zeithaml, 1991). Bajo este mismo aspecto se identifica la importancia del valoren los constructos clave de la calidad del servicio, además de su relación con la calidad percibida que compara la excelencia o superioridad relativa del producto o
66 ... la medición de valor para el cliente se relaciona con el conocimiento aceptado colectivamente, más

exactamente con la concepción de valor en el mercado en donde aparecen modelos que brindan información, explicación y clasificación de lo que más valoran los clientes; entre estos encontramos el valor monetario visto desde una perspectiva técnica, económica, del servicio y de los beneficios sociales que el cliente puede recibir a cambio del precio que paga. 
66 ... dentro de este ámbito se ha evidenciado el interés de la creación de valor experiencial de consumo para convertirla en una fuerte ventaja competitiva, yendo más allá de elementos funcionales y utilitaristas insertando aspectos más ambientales de carácter emocional o bedonista. servicio que el consumidor ve como sustituto, por lo que es importante el estudio de las relaciones de causalidad (Duque, 2005).

De acuerdo con Montaña (2004) y Naranjo (2000), otra concepción importante es el valor de marca, ya que este puede y debe ser el factor determinante, diferencial e inimitable para crear o agregar valor a productos, servicios y empresas, donde según los autores, su acertada gestión se fundamenta y logra su función en las dimensiones que conforman la equidad de marca (Montaña, 2004; Naranjo, 2000). En este mismo sentido el valor de marca es significativamente positivo con relación a precios y retornos, ya que es posible estimar la rentabilidad por el reconocimiento de la misma así no tenga inferencia con la percepción del valor para el mercado, pues asocia el gasto publicitario y las acciones en el mercado (Barth, Clement, Foster y Kasznik, 1998; Slater y Narver, 1994).

Ahora bien, las investigaciones acerca del consumo han influenciado la búsqueda de nuevos aspectos que infieren la determinación de valor. Para Holbrook y Gardner (1993), una de esas inferencias es el valor emocional, ya que este determina el lapso de tiempo que el cliente se toma para disfrutar la experiencia de consumo generado por el placer sensorial que algún estímulo pueda llegar a promover (Holbrook y Gardner, 1993). También dentro de este ámbito se ha evidenciado el interés de la creación de valor experiencial de consumo para convertirla en una fuerte ventaja competitiva, yendo más allá de elementos funcionales y utilitaristas insertando aspectos más ambientales de carácter emocional o hedonista (Sánchez-Fernández e Iniesta-Bonillo, 2009).

Demostrando la complejidad del concepto de valor, continúa en aumento la generación de nuevos conceptos. Recientemente se han desarrollado nuevas perspectivas del valor como: el valor superior, el valor del cliente hacia la firma, el valor percibido del consumidor, entre otros ejemplos (Payne y Holt, 2001), que suelen incidir en la dinamicidad del concepto, proponiendo valores anteriores y posteriores a la compra (Woodruff, 1997), o enriqueciendo su multidimensionalidad (J. N. Sheth, Newman y Gross, 1991). 


\section{PILARES CONCEPTUALES DEL VALOR DESDE EL MARKETING}

En los conceptos de valor ya mencionados se identifica la continua aparición de cuatro nociones: la satisfacción, la calidad, la lealtad y el precio. Gallarza, Gil y Holbrook (2011) realizan una investigación al respecto en donde se evidencia la interrelación de estos términos y cómo generan influencias en el comportamiento de los consumidores.

Dicha interrelación se muestra en la Figura 2, donde se muestran los vínculos identificados por los autores.

\section{Figura 2. Vínculos entre los constructos} relacionados con el valor. Una revisión.

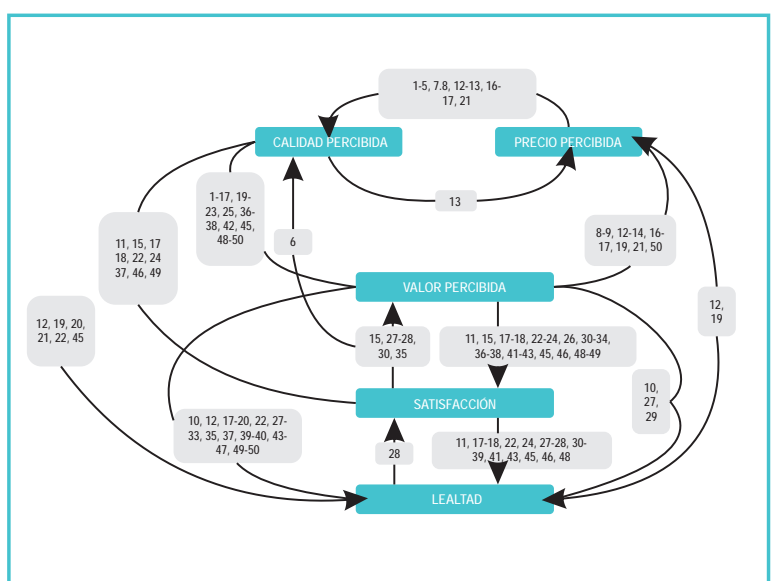

Fuente: Gallarza et al., 2011
Según la investigación realizada, se identifica una interconexión entre el valor percibido, la satisfacción y la lealtad con la calidad percibida, mientras que el precio se vincula con la lealtad, el valor y la calidad, mas no con la satisfacción. Asimismo, identifica cronológicamente a los autores que plantean dichas relaciones (véase la Tabla 2).

De acuerdo con la Tabla y la Figura 2, por ejemplo, Grenwal, Krishnan et al. (1998) identificaron los lazos entre la calidad percibida y el precio percibido, que luego Sirohi et al. (1998) y Kashyap y Bojanic (2000) relacionaron con la lealtad.

Por otro lado, en un nivel más macro Holbrook, Sánchez e Iniesta (2009) proponen una tipología del valor para el consumidor fundamentada en 4 conceptos: lo económico, lo social, lo altruísta y lo hedónico, como muestra la Tabla 3.

En esta tabla se aprecia la agrupación de los valores extrínsecos relacionados con la eficiencia y la excelencia -asociada a la calidad- como parte del valor económico, mientras que el estatus y la estima pertenecen al valor social. De igual,modo, valores intrínsecos como el juego y la estética se agrupan como valor hedónico

Tabla 2. Autores de los vínculos entre los constructos relacionados con el valor. Una revisión.

\begin{tabular}{|l|l|}
\hline 1. Monroe \& Krishnan (1985) & 26. Babin \& Kim (2001) \\
\hline 2. Doods \& Monroe (1985) & 27. Petrick et al. (2002) \\
\hline 3. Monroe \& Chapman (1987) & 28. Petrick \& Backman (2002) \\
\hline 4. Zeithaml (1988) & 29. Oh (2003) \\
\hline 5. Doods et al. (1991) & 30. Chiou (2004) \\
\hline 6. Bolton \& Drew (1991) & 31. Yang \& Peterson (2004) \\
\hline 7. Monroe (1992) & 32. Duman \& Mattila (2005) \\
\hline 8. Li et al. (1994) & 33. Grace \& O'Cass (2005) \\
\hline 9. Chang \& Wildt (1994) & 34. Lin et al. (2005) \\
\hline 10. Wakefield \& Barnes (1996) & 35. Lin \& Wang (2006) \\
\hline 11. Fornell et al. (1996) & 36. Gallarza \& Gil (2006) \\
\hline 12. Sirohi et al. (1998) & 37. Um et al. (2006) \\
\hline
\end{tabular}




\begin{tabular}{|l|l|}
\hline 13. Grewal, Krishnan et al. (1998) & 38. Chen \& Tsai (2007) \\
\hline 14. Grewal et al (1998) & 39. Lee et al. (2007) \\
\hline 15. Oliver(1999) & 40. Feng \& Morrison (2007) \\
\hline 16. Sweeny et al. (1999) & 41. Carpenter (2008) \\
\hline 17. Oh (1999) & 42. Yuan \& Wu (2008) \\
\hline 18.Tam(2000) & 43. Ryu et al. (2008) \\
\hline 19. Kashyap \& Bojanic (2000) & 44. Chen\&Tsai (2008) \\
\hline 20. Murphy et al. (2000) & 45. Hutchinson et al. (2009) \\
\hline 21. Oh (2000) & 46. He \& Song (2009) \\
\hline 22. Cronin et al. (2000) & 47. Cho \& Shiu (2009) \\
\hline 23. Caruana et al. (2000) & 48. Gallarza et al. (2009) \\
\hline 24. McDougall \& Levesque (2000) & 49. Hu et al. (2009) \\
\hline 25. Teas \& Agarwal (2000) & 50. Brodie et al. (2009) \\
\hline
\end{tabular}

Fuente: Gallarza et al., 2011.

Tabla 3. Tipología del valor para el consumidor.

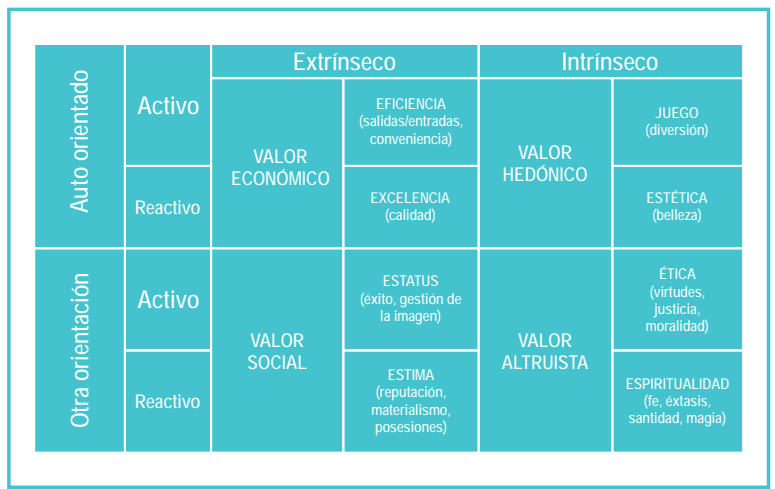

Fuente: Holbrook et al., 2009.

y, finalmente, la ética y la espiritualidad forman parte del valor altruísta.

De acuerdo con estas dos propuestas, en la Figura 3 se aprecian los pilares conceptuales del valor para el marketing, los cuales se establecen de la interrelación entre la satisfacción, la lealtad, el precio y la calidad con la agrupación de los valores económicos, sociales, hedónicos y altruístas.

Figura 3. Pilares conceptuales del valor en el marketing.

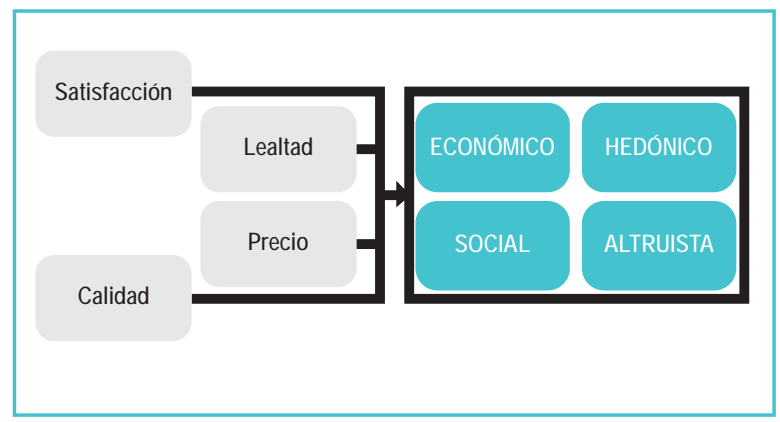

Fuente: elaboración propia.

\section{CONCEPTO dE VALOR DESDE El DISEÑO}

La investigación de valor en el diseño ha sido en gran medida dejada de lado, por lo que no se han desarrollado enfoques para su estudio, así que a continuación se presentarán los conceptos explorados según su grado de importancia (reiteración e incidencia).
El concepto con mayor asociación en el campo del diseño es el valor formal del producto, donde autores como Bloch (1995), Ulrich y Ellison (1999), proponen que las respuestas del consumidor a la forma del producto son variables, lo cual diferencia qué metas de diseño y qué 
constructos desarrollan una gran influencia en la configuración formal del producto; pero eso no es todo, además plantean que existen influencias de los factores situacionales y gustos individuales, los cuales son determinantes en la respuesta psicológica -cognitiva y afectiva- que termina reflejándose en la respuesta comportamental del consumidor (Bloch, 1995; K. Ulrich y Ellison, 1999). De la misma manera, la valoración del proceso de creación de valor delega mayor importancia, ya que acarrea significado para el consumidor, donde el valor no es solo un fenómeno sino un aspecto multifacético por considerar, visto a tal punto de ser un sistema de valor dentro de la organización (Bowman y Ambrosini, 2010; Carson, Devinney, Dowling y John, 1999).

Para comprender el intrincado sistema de diseño de producto es necesario generar un proceso de integración de conceptos presentado en la Figura 4, la cual fusiona el modelo creado por Bloch (1995, p. 17), y el modelo identificado por Holbrook y Hirschman (1982, p. 133), que nos muestra un panorama más amplio en la correspondencia del comportamiento del consumidor con relación a la forma del producto y la experiencia, aspecto de vital importancia para conjugar los conceptos y sintetizar la variedad de los mismos.

En esta integración podemos identificar que las metas y constructos de diseño, así como las influencias del contexto y del consumidor, tienen una relevancia considerable para la determinación de la forma del producto, pero luego de otorgarle forma el sistema de respuesta del consumidor no solo asocia a la forma sino a todo ese trasfondo que la originó, procesando dicha información basada en criterios y aprendizaje previamente identificados, generando como resultado un comportamiento afín a toda esta interacción.

El valor funcional es otra concepción asociada al diseño, debido a que los aspectos de uso están ligados a la relación de los deseos del consumidor y a los costos de producción (Jonas, 2001; Otto y Wood, 2000), pero sobre todo a la utilidad que

\section{${ }^{66}$ El valor funcional es otra concepción asociada al diseño, debido a que los aspectos de uso están ligados a la relación de los deseos del consumidory a los costos de producción ... , pero sobre todo a la utilidad que pueda brindar el producto o servicio.}



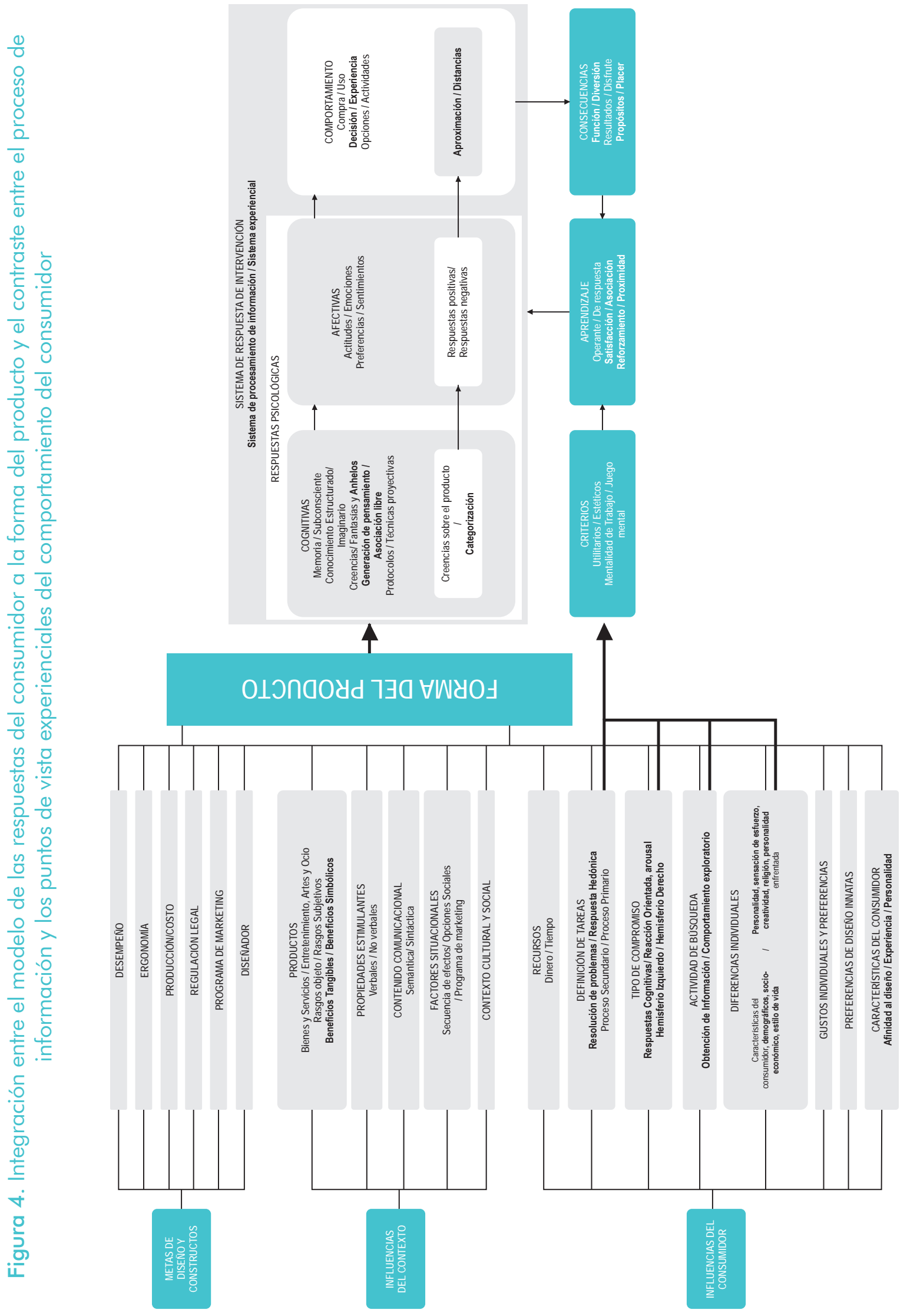

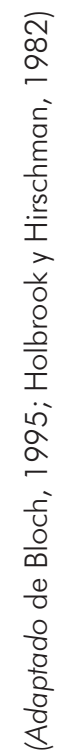


pueda brindar el producto o servicio. De igual manera, esto va relacionado con la generación de un valor integral de producto que con ayuda de una comunicación adecuada y clara establece una experiencia de diseño diferente para cada consumidor (Maeda, 2006).

En torno al diseño es importante delimitar aspectos como el gusto, la apariencia y demás características que se incluyen en el valor del producto, llamado el valor estético, lo que permite orientar el concepto de diseño y establecer relaciones de diferenciación y posicionamiento con los clientes o consumidores, o bien de reducción de costos para las organizaciones (Desai, Kekre, Radhakrishnan y Srinivasan, 2001; Montaña, 1991; Veryzer y Borja de Mozota, 2005).

Por su parte Holbrook, Sánchez e Iniesta (2009) afirman que sin mucha socialización se ha tratado de medir la respuesta estética del consumidor a las características del diseño, donde variables como la visualización y verbalización de la tendencia, la motivación intrínseca y extrínseca, el romanticismo y clasicismo, así como el sexo, pero enfocado al arte y a la moda, serían aspectos por medir, por más que sus características abstractas hagan difícil su definición y evaluación (Holbrook et al., 2009). Pero al existir muchas más variables que no se han tenido en cuenta, estos parámetros quedarían algo cortos al hablar del diseño industrial, gráfico, de interiores y demás.

De igual manera, la aparición de valores psicológicos relacionados con el desempeño de los objetos cotidianos que buscan comprender, interpretar y lógicamente manipular estos objetos de manera intuitiva, representan una diferencia positiva en la calidad de vida de la sociedad. Según Norman (1990), algunos de estos conceptos son: prestaciones, retroalimentación, modelo conceptual adecuado, topografía, visibilidad, seguridad, tecnología, por mencionar algunos, los cuales buscan evitar la invisibilidad, la arbitrariedad, la incoherencia, la no inteligibilidad, la descortesía, las limitaciones, la deliberada dificultad, lo peligroso y lo excesivamente complejo (Norman, 1990).
La medición del valor conceptual concibe mayor relevancia en la investigación y desarrollo de producto, ya que permite verificar los problemas de diseño que se pudieran presentar más allá de los resultados costo/beneficio, lo que denominan en su modelo Dahan y Mendelson valor extremo, donde analizan los roles y parámetros de la distribución de la ganancia, así como el costo de determinados análisis en comparación con el presupuesto para el diseño del concepto dentro de las fases de desarrollo (Dahan y Mendelson, 2001).

Con la intención de fomentar la utilidad, influir en la percepción e incrementar el atractivo de los productos, así como de aumentar el valor para el cliente, surgen los denominados impulsores de valor o valores relacionales, los cuales se basan en la relación estéticautilidad, flexibilidad-eficacia y costo-beneficio, agregando aspectos como la accesibilidad, la adecuación, el condicionamiento emocional, la disonancia cognitiva, el color, la comparación, la limitación y demás principios afines (Lidwell, Holden y Butler, 2005).

El valor diferencial surge a partir del proceso de diseño, el cual genera una respuesta innovadora, creativa y nueva, que se destaque en el mercado y provea el atractivo suficiente para cumplir con las necesidades y requerimientos del consumidor, pero no es otorgado o insertado, es más bien innato de cada diseño ya que "el valor intrínseco del producto tiene que ser percibido por el consumidor o el usuario, por ello el diseño se ocupa de hacerlo visible dándole la forma más adecuada y aplicando la comunicación más efectiva" (Viladas, 2010, p. 36).

No puede negarse que la existencia de valores individuales permite la personalización y enriquecimiento del diseño, pero su inserción y formalización dependen de su aceptación colectiva y de la crisis de estos en la actualidad donde se han roto valores compartidos como los nacionales, tribales, personales y hasta los familiares. Igarashi, Medina, Brattinga y Baur 
(1993), plantean que la existencia de nuevos valores consensuales que crean un sentido común y nuevos propósitos, apareciendo conceptos como calidad, utilidad, funcionalidad, responsabilidad (ética, moral, ambiental, etc.), actualidad, belleza, versatilidad, entre otras (lgarashi, Medina, Brattinga y Baur, 1993).
Dentro de valor del producto también hay aspectos para la optimización del proceso de diseño, en este caso el valor del tiempo en donde, debido a la integración, se reduzcan costos de manufactura y se brinde el tiempo necesario a las actividades que más lo requieran (Jones, 1982; K. Ulrich, Sartorius, Pearson y Jakiela, 1993).

\section{PILARES CONCEPTUALES DEL VALOR DESDE EL DISEÑO}

A partir de la revisión realizada se evidencia que existen tres grandes nociones conceptuales asociadas al diseño: lo formal, lo útil y lo funcional. Estos tres conceptos tienden a ser repetitivos aunque sus características sí suelen ser clasificadas en forma variada.

Es claro que no son los únicos patrones por seguir, ya que autores como Bonsiepe (1993), Gómez-
Senent Martínez y Capuz Rizo (1999), Munari y Rodríguez (1989), Pugh (1991), y Rodríguez (1997), han planteado varias clasificaciones de los criterios de diseño, manejando como fundamentos conceptuales (además de los 3 ya mencionados), lo estructural, lo técnico/productivo, de identificación, económicos y del mercado, así como los legales (remítase a la Tabla 4), los cuales podrían ser aplicables de igual manera al concepto de valor.

\section{Tabla 4. Requerimientos que se valoran en el diseño.}
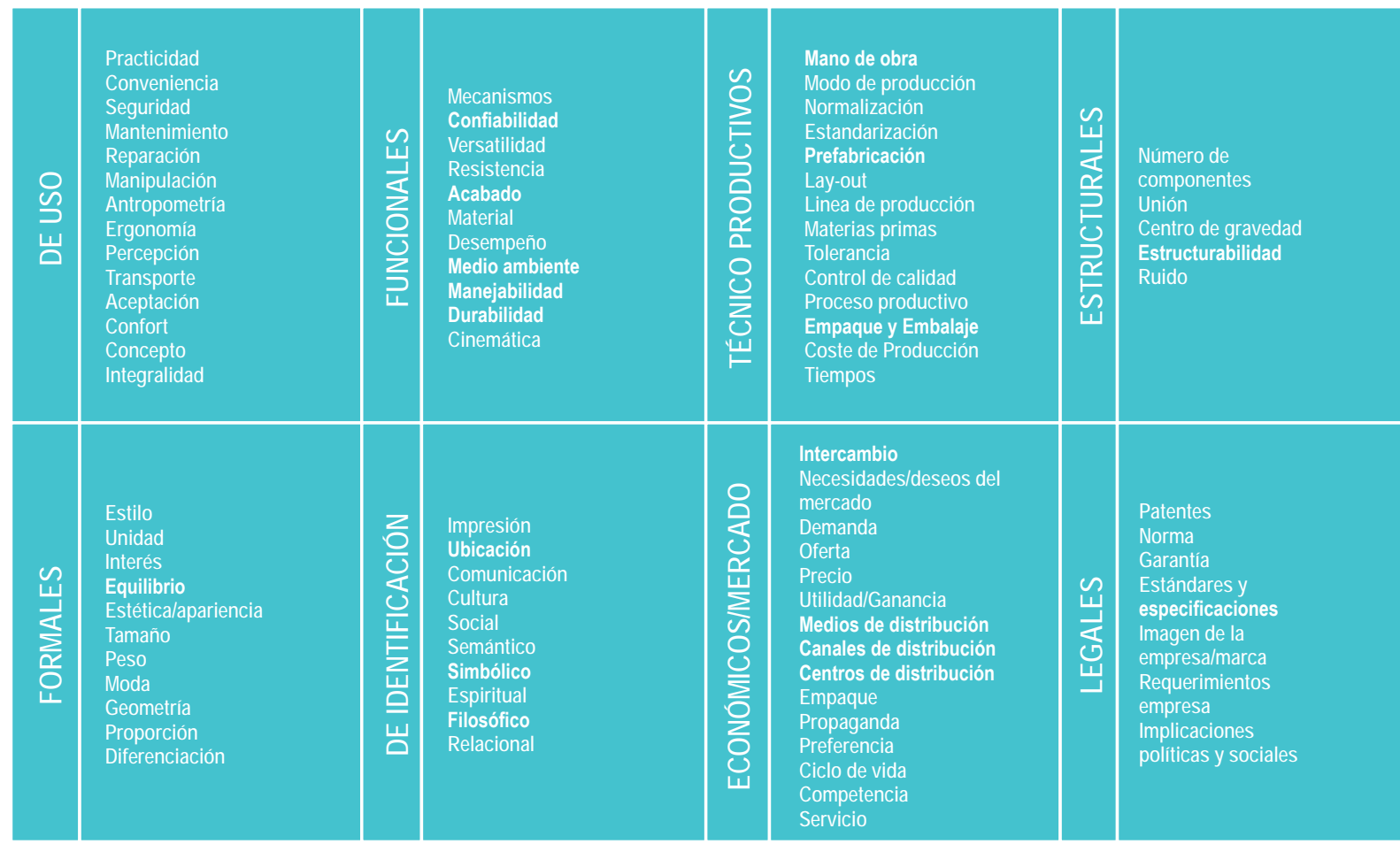

(Adaptado de Bonsiepe, 1993; Gómez-Senent Martínez y Capuz Rizo, 1999; Munari y Rodríguez, 1989; Pugh, 1991; Rodríguez, 1997) 
De acuerdo con lo anterior, en la Figura 5 apreciamos la síntesis de los pilares conceptuales del valor para el diseño.

Figura 5. Pilares conceptuales del valor en el diseño.

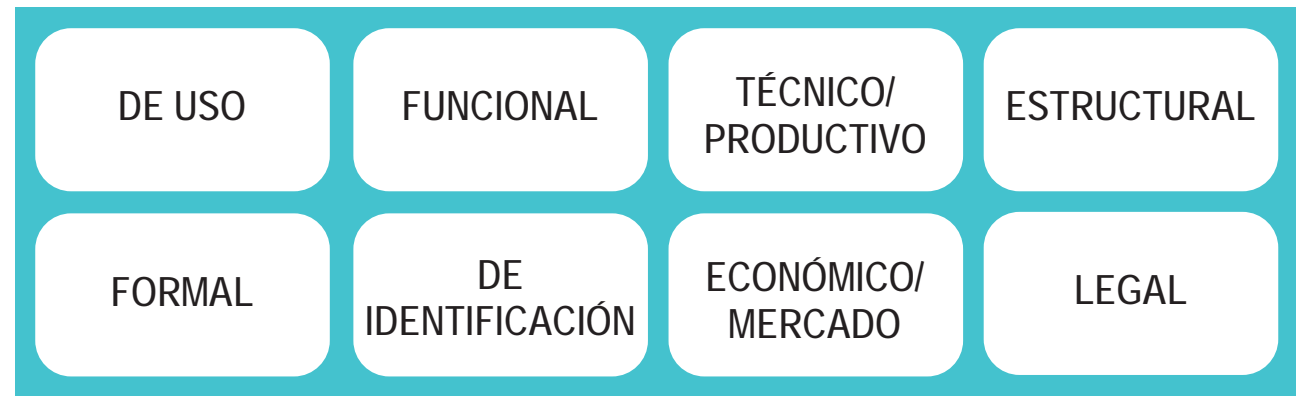

Fuente: elaboración propia.

\section{ANÁLISIS DEL CONCEPTO DE VALOR CONJUNTO ENTRE - LAS DOS DISCIPLINAS}

En primera medida, para este análisis es necesario tener en cuenta que la cantidad de información no es la misma, es indiscutible que las investigaciones sobre valor en marketing han aumentado y son constantemente publicadas, mientras que en el diseño es difícil encontrar investigaciones precisas sobre esta temática $y$, aún peor, suelen quedarse como conocimiento tácito dentro de las universidades.

Segundo, es indudable la influencia de otras disciplinas tanto para el marketing como para el diseño, pero no es claro el por qué de la diferenciación entre ellos, es decir, al diseño se le relaciona con la artes, la psicología, lo social, lo humano y lo ambiental, pero casi nunca tiende a ser asociado con lo económico o administrativo, por más que también tienen mucho en común.

Y tercero, la existencia de valores que están supeditados a otros hace que sea más complejo el estudio y la interrelación entre ellos, pues la concepción de estos permea los valores individuales y compartidos, modificándolos y cambiando su uso en la toma de decisión.
Ya identificados los pilares conceptuales para el marketing y el diseño es posible compararlos bajo los criterios de significación, asociación e interconexión, donde podría generarse una relación como se muestra en la figura 6, la cual integra los conceptos afines y se muestra su interrelación entre sí.

Figura 6. Integración pilares conceptuales del valor entre el marketing y el diseño.

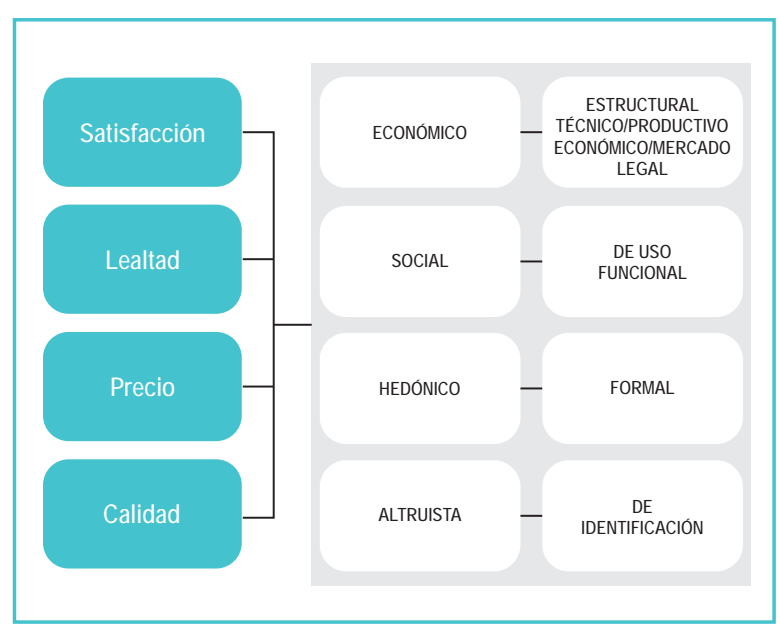

Fuente: Elaboración propia. 
La aparición de vínculos claros entre cada uno de los pilares conceptuales del valor entre el marketing y el diseño se hace de manera intuitiva gracias a la clasificación antes presentada, donde se enlaza lo económico con lo estructural, técnico/productivo, legal y del mercado, así como lo social con lo funcional y de uso, lo hedónico con lo formal, y lo altruísta con el pilar de identificación. Como se mostró anteriormente en la Figura 3, existe una asociación entre la satisfacción, lealtad, precio y calidad y los pilares del marketing, así que esta conexión se mantendría para los de diseño debido a los vínculos ya mencionados.

Aunque esta propuesta de integración permite demostrar la interconexión de los conceptos de valor, particularmente en el diseño sí existen diferencias en la manera de priorizar y usar algunos valores. En el ámbito académico y sin mayor sustentación investigativa, existen desacuerdos en el uso de ciertos términos, por ejemplo, al hablar de valor hedonista, que normalmente es asociado al placer, no tendría mayor relación con el juego y la estética tal como sucede con el marketing. Además, el valor de marca para el diseñador (exceptuando al gráfico), pierde relevancia sobre otros valores que considera de mayor jerarquía como el valor funcional, experiencial, ambiental, innovador, comunicativo, comportamental y del servicio. Lo mismo le sucede al valor de intercambio, el cual pierde importancia sobre los otros.

Un aspecto que no se ha trabajado como valor pero es una característica infaltable para las dos disciplinas es la innovación; para llegar a ella se requiere un proceso de investigación y desarrollo, el cual utiliza y se apoya en el conocimiento actual para producir nuevo conocimiento aplicado a procesos, productos, entre otros, definiéndose entonces como la "inserción de valor" en el mercado, pero para ello debe ser claro -para nuestro caso- el know what, know why, know how y el know who' del conocimiento del marketing y del diseño (Gallego, 2007).

Finalmente, no hay que dejar de lado la importancia que pueden tener los valores personales innatos en el ser humano para analizar el comportamiento del consumidor, ya que estos tienen implicaciones e influencias en el momento de escoger marcas, productos y atributos (Vinson, Scott y Lamont, 1977).

\section{CONCLUSIONES, REFLEXIONES Y OPORTUNIDADES SOBRE 8. EL ESTUDIO DEL CONCEPTO DE VALOR PARA EL MARKETING Y EL DISEÑO}

La caracterización de los conceptos de valor permitió tener un poco más de claridad sobre las diferentes concepciones de valor para las dos disciplinas, ya que permitió generar una clasificación de valores, así como una propuesta de integración de sus pilares conceptuales, los cuales buscan contribuir de alguna manera a la investigación de valor para el marketing y el diseño.

El saber qué, saber por qué, saber cómo y saber quién.
Esta identificación y clasificación permitió conocer la amplia gama de valores que se desarrollan en cada una de las disciplinas, mostrando sus compatibilidades y diferencias, las cuales facilitaron el desarrollo de un análisis comparativo que determinó el grado de articulación entre ellas.

Considerando esta revisión es evidente que existe una pequeña brecha entre el concepto de 
valor en el marketing y el diseño, dadas algunas diferencias conceptuales, pero sobre todo por el grado de importancia que se le da a cada una, pues aunque de manera general tienen mucho en común, el desconocimiento, la falta de investigación y comunicación al respecto han hecho que muchas veces se generen incongruencias que fomentan el choque entre las dos disciplinas.

Es evidente que la desconexión entre las dos disciplinas hace que los encargados del marketing muchas veces tengan más claros los valores de diseño que los mismos diseñadores (Duarte, 2008), problema muy perjudicial para la organización, por lo cual la gestión de diseño y marketing es completamente necesaria.

Por el lado del marketing existe un estudio juicioso del concepto de valor, pero al dejar de lado conceptos manejados por el diseño, está limitando su alcance y sus futuros usos. En cuanto al diseño, al no existir variada literatura sobre el estudio del valor en el diseño en comparación con el marketing, se hace necesario recurrir a la información que tienen los docentes.

Del mismo modo, desde el diseño aparecen otros valores de vital importancia como el valor de uso, cultural, antropológico, filosófico, histórico, tecnológico, científico, semántico y simbólico (Álvarez y Martínez, 2009), los cuales tienen un potencial de investigación enorme aún no desarrollado por el marketing.

En cuanto a las oportunidades, una de las ventajas de esta reflexión es proveer un marco conceptual articulado del valor visto desde el marketing y el diseño, donde su futura contribución estaría en contrastación en el mundo real, visto desde varias perspectivas: primero, logrando agrupar un panel de expertos para construir un experimento que ponga prueba dicho marco y, además, brinde información para la creación de un modelo para el marketing y el diseño; por otro lado, puede evaluarse desde el punto de vista académico haciéndolo parte de un programa o proyecto investigativo en donde se pruebe su concepción
${ }^{6}$ Por el lado del marketing existe un estudio juicioso del concepto de valor, pero al dejar de lado conceptos manejados por el diseño, está limitando su alcance y sus futuros usos. En cuanto al diseño, al no existir variada literatura sobre el estudio del valor en el diseño en comparación con el marketing, se hace necesario recurrir a la información que tienen los docentes. 
y aplicación; y por último, también podría contrastarse dentro de una organización para mostrar su congruencia en el mundo empresarial.

Podría también aplicarse en el área del neuromarketing, donde sería posible generar investigaciones y pruebas que conjuguen las dos disciplinas y brinden información pertinente para la toma de decisiones de marketing y diseño (Ariely y Berns, 2010; Fugate, 2008; Lee, Broderick y Chamberlain, 2007; Renvoisé y Morin, 2007).

\section{BIBLIOGRAFÍA}

Álvarez, F. A., \& Martínez, E. E. (2009). Consideraciones para un enfoque complejo y sistémico de las competencias en y para la innovación social. Universidad Jorge Tadeo Lozano. Bogotá, Colombia.

Anderson, J., \& Narus, J. (1998). Business marketing: understand what customers value. Harvard business review, 76, 53-67. Boston, USA.

Ariely, D., \& Berns, G. S. (2010). Neuromarketing: the hope and hype of neuroimaging in business. Nature reviews. Neuroscience, 17 (4), 284.New York, USA.

Barth, M., Clement, M., Foster, G., \& Kasznik, R. (1998). Brand values and capital market valuation. Review of accounting studies, 3(1), 41-68. Berkeley, USA

Berger, P., \& Nasr, N. (1998). Customer lifetime value: Marketing models and applications. Journal of Interactive Marketing, 12(1), 17 30. London, UK.

Berry, L. (1995). Relationship marketing of services - growing interest, emerging perspectives. Journal of the Academy of Marketing Science, 23(4), 236-245. Ruston, USA

Bloch, P. (1995). Seeking the ideal form: Product design and consumer response. The Journal of Marketing, 59(3), 16-29. Chicago, USA.

Bonsiepe, G. (1993). Las 7 columnas del diseño. Editorial UAM-Unidad Azcapotzalco, 1. Axcapotzalco, Mexico.
Borle, S., Singh, S., \& Jain, D. (2008). Customer lifetime value measurement. Management science, 54(1), 100. Hanover, USA.

Bowman, C., \& Ambrosini, V. (2010). How value is created, captured and destroyed. European Business Review, 22(5), 479-495.

Brown, T. (2009). Change by design: how design thinking transforms organizations and inspires innovation: HarperBusiness. New York, USA.

Bruce, M., \& Daly, L. (2007). Design and marketing connections: creating added value. Journal of marketing management, 23(9-10), 929-953. Morehead, USA.

Carson, S., Devinney, T., Dowling, G., \& John, G. (1999). Understanding institutional designs within marketing value systems. The Journal of Marketing, 63, 115-130. Chicago, USA.

Christopher, M. (1991). Relationship marketing: bringing quality customer service and marketing together. Butterworth Heinemann. Oxford, UK.

Cronin Jr, J., Brady, M., \& Hult, G. (2000). Assessing the effects of quality, value, and customer satisfaction on consumer behavioral intentions in service environments. Journal of retailing, 76(2), 193-218. Tucson, USA.

Dahan, E., \& Mendelson, H. (2001). An extremevalue model of concept testing. Management science, 47(1), 102-116. Hanover, USA. 
Desai, P., Kekre, S., Radhakrishnan, S., \& Srinivasan, K. (2001). Product differentiation and commonality in design: Balancing revenue and cost drivers. Management science, 47(1), 37-51. Hanover, USA.

Duarte, N. (2008). Slideology: The Art and Science of Presentation Design. Taschenbuch, O'Reilly Media, 978-0596522346. Sebastopol, USA.

Duque, E. (2005). Revisión del concepto de calidad del servicio y sus modelos de medición. Revista Innovar, 15, 25. Bogotá, Colombia

Fugate, D. L. (2008). Marketing services more effectively with neuromarketing research: a look into the fu

Gallarza, M. G., Gil Saura, I., \& Holbrook, M. B. (2011). The value of value: Further excursions on the meaning and role of customer value. Journal of Consumer Behaviour, 10(4), 179 . 191.

Gallego, J. (2007). Economía del conocimiento e innovación. In M. Palacio, R. Domínguez \& H. Cardona (Eds.), Ética, Innovación y Estética. Instituto tecnológico metropolitano. Medellín, Colombia.

Gil, I., \& Gallarza, M. G. (2008). La investigación en valor percibido desde el marketing. Innovar. Revista de Ciencias Administrativas y Sociales(031), 9-17. Bogotá, Colombia.

Gil, I., \& Molina, M. E. R. (2009). Valor de la relación, compromiso, TIC y lealtad en el canal de comercialización. Innovar. Revista de Ciencias Administrativas y Sociales(33), 77 90. Bogotá, Colombia

Glosten, L., Jagannathan, R., \& Runkle, D. (1993). On the relation between the expected value and the volatility of the nominal excess return on stocks. Journal of finance, 48(5), 1779-1801. Aldan, USA.
Gómez-Senent Martínez, E., \& Capuz Rizo, S. (1999). El proyecto y su dirección y gestión. Universidad Politécnica de Valencia. Valencia, España.

Grönroos, C. (1997a). Keynote paper From marketing mix to relationship marketingtowards a paradigm shift in marketing. Management decision, 35(4), 322-339.

Grönroos, C. (1997b). Value-driven relational marketing: from products to resources and competencies. Journal of marketing management, 13(5), 407-419. Scotland, UK.

Grönroos, C. (2004). The relationship marketing process: communication, interaction, dialogue, value. Journal of Business \& Industrial Marketing, 19(2), 99-113.

Gupta, S., Hanssens, D., Hardie, B., Kahn, W., Kumar, V., Lin, N., et al. (2006). Modeling customer lifetime value. Journal of Service Research, 9(2), 139. Haryana, India

Hogan, J. (2001). Expected Relationship Value:: A Construct, a Methodology for Measurement, and a Modeling Technique. Industrial Marketing Management, 30(4), 339-351. Vernon, USA.

Holbrook, M. (1996). Special session summary customer value a framework for analysis and research. Advances in Consumer Research, 23, 138-142. Duluth, USA.

Holbrook, M., \& Gardner, M. (1993). An approach to investigating the emotional determinants of consumption durations: why do people consume what they consume for as long as they consume it? Journal of Consumer Psychology, 2(2), 123-142.New York, USA.

Holbrook, M., \& Hirschman, E. (1982). The experiential aspects of consumption: Consumer fantasies, feelings, and fun. Journal of consumer research, 9(2), 132-140. Madison, USA 
Holbrook, M., Sánchez, R., \& Iniesta, M. (2009). The conceptualisation and measurement of consumer value in services. International Journal of Market Research, 51(1), 93-113. Oxford, UK.

Igarashi, T., Medina, F., Brattinga, P., \& Baur, R. (1993). Mi relación con el Diseño; El diseño, una vision global; El diseño en un mundo finito; Diseño por substracción. In M. Senestrari (Ed.), Ensayos sobre Diseño. Ediciones Infinito. Buenos Aires, Argentina.

Jain, D., \& Singh, S. (2002). Customer lifetime value research in marketing: A review and future directions. Journal of Interactive Marketing, 16(2), 34-46. London, UK.

Jonas, W. (2001). A scenario for design. Design issues, 17(2), 64-80. Cambridge, USA

Jones, C. (1982). Métodos de Diseño.Editorial Gustavo Gili. Barcelona, España.

Lee, N., Broderick, A. J., \& Chamberlain, L. (2007). What is neuromarketing? A discussion and agenda for future research. International Journal of Psychophysiology, 63(2), 199-204.

Lidwell, W., Holden, K., \& Butler, J. (2005). Principios Universales de Diseño. Blume. Barcelona, España.

Llompart, R. (2009). La importancia del Diseño de Comunicación Visual desde las enseñanzas artísticas medias y superiores. Paper presented at the Encuentro Latinoamericano de Diseño. Palermo, Argentina.

Maeda, J. (2006). The laws of simplicity: The MIT Press. Cambridge, USA.

Mele, C. (2007). The synergic relationship between TQM and marketing in creating customer value. Managing Service Quality, 17(3), 240-258.
Monroe, K. B. (1979). Price: Making profitable decisions.McGraw-Hill. New York, USA.

Montaña, J. (1991). Diseño de nuevos productos. Temes de disseny, 12. Barcelona, España.

Montaña, J. (2004). Design management in the creation of brands.Barcelona, España

Montaña, J., \& Fundación, B. (2001). Diseño: Rentabilidad social y rentabilidad económica. Ministerio de Ciencia y Tecnología. Barcelona, España.

Morgan, R., \& Hunt, S. (1994). The commitmenttrust theory of relationship marketing. The Journal of Marketing, 58(3), 20-38. Chicago, USA.

Munari, B., \& Rodríguez, C. A. (1989). ¿Cómo nacen los objetos? : Gustavo Gili. Barcelona, España

Naranjo, S. (2000). La equidad de marca "brand equity" una estrategia para crear y agregar valor. Estudios Gerenciales. Cali, Colombia.

Norman, D. (1990). La Psicología de los objetos cotidianos. Editorial Nerea. Madrid, España.

Otto, K. N., \& Wood, K. L. (2000). Product design: Prentice Hall Englewood Cliffs, New Jersey.USA

Parasuraman, A. (1997). Reflections on gaining competitive advantage through customer value. Journal of the Academy of Marketing Science, 25(2), 154-161. Ruston, USA.

Parasuraman, A., Berry, L., \& Zeithaml, V. (1991). Understanding customer expectations of service. Sloan Management Review, 32(3), 39-48. Cambridge, USA.

Payne, A., \& Holt, S. (2001). Diagnosing customer value: integrating the value process and relationship marketing. British Journal of Management, 12(2), 159-182. UK. 
Pugh, S. (1991). Total design: integrated methods for successful product engineering: Wokingham, England.

Ravald, A., \& Grönroos, C. (1996). The value concept and relationship marketing. European Journal of Marketing, 30(2), 19-30.

Renvoisé, P., \& Morin, C. (2007). Neuromarketing: Understanding the Buy Buttons in Your Customer's Brain. Sales Brain LLC, Nashville, USA.

Rodríguez, G. (1997). Manual de diseño industrial: Gustavo Gili , DF, Mexico.

Sanchez-Fernandez, R., \& Iniesta-Bonillo, M. (2006). Consumer perception of value: Literature review and a new conceptual framework. Journal of Consumer Satisfaction Dissatisfaction and Complaining Behavior, 19, 40. La Verne, USA.

Sánchez-Fernandez, R., \& Iniesta-Bonillo, M. (2009). La estética y la diversión como factores generadores de valor en la experiencia de consumo en servicios. Revista Innovar, 19. Bogotá, Colombia.

Sheth, J., \& Parvatiyar, A. (1995). Relationship marketing in consumer markets: antecedents and consequences. Journal of the Academy of Marketing Science, 23(4), 255-271. Ruston, USA.

Sheth, J. N., Newman, B. I., \& Gross, B. L. (1991). Why we buy what we buy: a theory of consumption values. Journal of business research, 22(2), 159-170.Nr Reading, UK.

Slater, S., \& Narver, J. (1994). Market orientation, customer value, and superior performance. Business horizons, 37, 22-22. Bloomington, USA.

Turnbull, J. (2009). CustomerValue-in-Experience: Theoretical foundation and research agenda. ANZMAC, Sidney, Australia.
Ulrich, K., \& Ellison, D. (1999). Holistic customer requirements and the design-select decision. Management science, 45(5), 641-658. Hanover, USA.

Ulrich, K., \& Eppinger, S. (2009). Diseño y desarrollo de productos. DF, Mexico.

Ulrich, K., Sartorius, D., Pearson, S., \& Jakiela, M. (1993). Including the value of time in design-for-manufacturing decision making. Management science, 39(4), 429-447. Hanover, USA.

Venkatesan, R., \& Kumar, V. (2004). A customer lifetime value framework for customer selection and resource allocation strategy. Journal of Marketing, 68(4), 106-125. Chicago, USA.

Veryzer, R., \& Boria de Mozota, B. (2005). The Impact of User Oriented Design on New Product Development: An Examination of Fundamental Relationships*. Journal of Product innovation management, 22(2), 128143. Chicago, USA.

Viladas, X. (2010). Diseño Rentable: 10 temas a debate (Vol. 2): Dami Editorial. Barcelona, España

Vinson, D., Scott, J., \& Lamont, L. (1977). The role of personal values in marketing and consumer behavior. The Journal of Marketing, 41 (2), 44 50. Chicago, USA.

Woodruff, R. (1997). Customer value: the next source for competitive advantage. Journal of the Academy of Marketing Science, 25(2), 139-153. Ruston, USA.

Zeithaml, V. (1988). Consumer perceptions of price, quality, and value: a means-end model and synthesis of evidence. The Journal of Marketing, 2-22. Chicago, USA. 
